\title{
Increase of passive safety of vehicles due to temperature calculation of brake units
}

\author{
Belokurov Vladimir Petrovich \\ Voronezh State University of Forestry and Technologies \\ named after G.F. Morozov \\ Voronezh, Russia 394087, Russian Federation \\ E-mail: opbd_vglta@mail.ru \\ Korablev Ruslan Alexandrovich \\ Voronezh State University of Forestry and Technologies \\ named after G.F. Morozov \\ Voronezh, Russia 394087, Russian Federation \\ E-mail: korablev_ruslan@mail.ru
}

\author{
Busarin Eduard Nikolaevich \\ Voronezh State University of Forestry and Technologies \\ named after G.F. Morozov \\ Voronezh, Russia 394087, Russian Federation \\ E-mail: busarin.eduard@mail.ru \\ Artemov Alexander Yurievich \\ Voronezh State University of Forestry and Technologies \\ named after G.F. Morozov \\ Voronezh, Russia 394087, Russian Federation \\ E-mail: alexandrvrn@mail.ru
}

\author{
Spodarev Ruslan Alexandrovich \\ Voronezh State University of Forestry and Technologies \\ named after G.F. Morozov \\ Voronezh, Russia 394087, Russian Federation \\ E-mail: spruslan78@mail.ru
}

\begin{abstract}
The article considers conditions for increasing passive safety of vehicles due to the use of temperature calculation in friction pairs of brake units taking into account their active heat dissipation. This temperature calculation allows designers at the design stage of new brake units to determine their optimal configuration, required frictional surface of the friction brakes, taking into account their thermal stability in conditions of increased friction dynamics, which, ultimately, ensures passive safety of transport.
\end{abstract}

Keywords- passive safety, temperature, friction units, brakes, heat dissipation

\section{INTRODUCTION}

In recent years, quantity and quality of vehicles produced in various operations have increased significantly. The most important components of vehicles are friction units - brakes, on which operability and safety of their operation depends as a whole.

An important factor affecting efficiency of brake units is a friction temperature mode. Brake units of modern transport ensure conversion of significant values of mechanical energy of motion into thermal energy for a short period of time. Heat generated by friction in brake friction pairs has significant effect on reliability and durability of friction units, that is, on the passive safety of transport as a whole. With increasing temperature, coefficient of friction (its decrease) and increase in wear of friction surfaces occur. Therefore, when designing brake units, it is necessary to take into account thermal tension that occurs in friction pairs, as well as to provide structural measures aimed at thermal regime reducing. This is especially important for vehicles with increased friction dynamics of brake units.

\section{RESULTS AND DISCUSSION}

The reduction of thermal loads of brake units is actual one and requires calculation of thermal dynamics of friction of brake units especially when using energy-saturated vehicles.

The study of temperature modes of brake units is, until now, carried out, as a rule, when considering solution of heat conduction problems without taking into account heat exchange with environment, that is, without possible heat dissipation $(\partial t / \partial x=0)$.

In order to determine temperature field of friction material of the brake at any time, it is necessary to have data on temperature distribution at the initial time (the initial conditions) and to know the heat exchange condition between the surface of the working body and environment.

The differential heat equation that determines nonstationary temperature field of brake friction pairs, taking into account their heat dissipation, expresses the relationship between temperature change $t$ in time $\tau$ and its propagation in the friction material with respect to $x, y$ and $z$ coordinates and is described quite adequately by the differential FourierKirchhoff equation. We assume that there are no sources of heat other than heat flow that occurs in the friction zone, and 
the loss of heat through the ends of friction material is small. Then the heat equation from a three-dimensional one can be reduced to a one-dimensional equation and takes the form:

$$
\frac{\partial t(x, \tau)}{\partial \tau}=a \cdot \frac{\partial^{2} t(x, \tau)}{\partial x^{2}}
$$

where $a$ - coefficient of temperature diffusivity, $\mathrm{m}^{2} / \mathrm{s}$.

In this regard, we assume that before the brakes are switched on $(t=0)$, friction material and metal surface have the same temperature throughout their mass (initial conditions):

$$
t(x, 0)=t_{\text {aver }}=\text { const } .
$$

Boundary conditions are determined from the condition that heat exchange takes place both inside the friction lining with area $\mathrm{F}$ at time $\mathrm{t}$ and between the surface of brake unit and surrounding medium.

Then boundary conditions in our case when $x=0$ :

$$
\frac{\partial t(0, \tau)}{\partial x}=-\frac{\left(1-\alpha_{H F}\right) \cdot W_{H F}}{A_{a} \cdot \tau_{F} \cdot \lambda_{1}} \cdot \tau_{N}
$$

when $x=\delta$

$$
\frac{\partial t(\delta, \tau)}{\partial x}=-h\left[t(\delta, \tau)-t_{\text {aver }}\right]
$$

where $\alpha_{\text {T.п. }}$ - coefficient of distribution of heat flows between friction elements [1]; $W_{H F}$ - full friction work, $\mathbf{J}$; $\tau_{N}=\frac{N_{\tau}}{N_{\text {taver }}}=2\left(1-\frac{\tau}{\tau_{F}}\right)-$ time factor of frictional power [4,5]; $\tau, \tau_{F}$ - current and total friction time, s; $A_{a}$ - nominal friction surface area of brake pad, $\mathrm{m}^{2} ; N \tau_{\text {aver }}, N \tau$ - average and current value of frictional power, $\mathrm{W} ; \delta$ - thickness of friction pad, m; $h=\frac{\alpha}{\lambda}$ - thermal coefficient, $1 / \mathrm{m} ; \alpha$ - coefficient of heat transfer from the surface of brake unit, $\mathrm{W} / \mathrm{m}^{2} \cdot{ }^{\circ} \mathrm{C} ; \lambda$ coefficient of thermal conductivity of friction material, $\mathrm{W} / \mathrm{m} \cdot{ }^{\circ} \mathrm{C}$.

To solve differential heat equation (1) with initial (2) and boundary (3) conditions, we make some assumptions:

1. Temperature field of friction material is identical to the field of a single homogeneous body.

2. Parameters $\lambda, c$ and $\rho$ do not depend on temperature and pressure.

3. We solve thermal problem for a wall of friction material, which is less heat-resistant in a friction pair. In calculation taking into account heat exchange of the environment with heat exchange surface, reduced thickness of friction pad has been used $\delta$ :

$$
\delta=\delta_{\text {th.p. }}+\delta_{m . s .}
$$

where $\delta_{\text {th.p. }}, \delta_{\text {m.s. }}$ - actual thickness of friction pad and thickness of metal support surface, which, according to the thermal resistance, is reduced to the thickness of friction material.

To solve equation (1) under condition (2) and (3), we use the Laplace transformation with respect to one of the variables, for example, time $\tau$.

$$
L_{\tau}[t(x, \tau)]=\int_{0}^{\infty} t(x, \tau) \cdot \exp (-g \tau) d \tau=T(x, g)
$$

As a result of carrying out the Laplace transformation with respect to time $\tau$, we obtain the following equation $[2,3]$ :

$$
T(x, g)-\frac{t_{\text {aver }}}{g}=A \cdot \operatorname{ch} \sqrt{\frac{g}{a}} x+B \cdot \operatorname{sh} \sqrt{\frac{g}{a}} x .
$$

Constants A and B are determined from the boundary conditions for the images, which after the Laplace transformation are obtained as follows:

when $x=0$,

$T^{\prime}(0, g)=-Q\left(1-\frac{1}{g \tau_{T}}\right) \cdot \frac{1}{g}$

when $x=\delta$,

$T^{\prime}(\delta, g)=-h\left[T(\delta, g)-\frac{t_{a v e r}}{g}\right]$

when $\tau=0$,

$T(x, 0)=t_{\text {aver }} \cdot \frac{1}{g}$

where $Q=\frac{2\left(1-\alpha_{H F}\right) \cdot W_{H F}}{A_{\epsilon} \cdot \tau_{F} \cdot \lambda_{1}}$

When $\mathrm{x}=0$ from equation (6) and (7) we have:

$$
\begin{aligned}
& T^{\prime}(0, g)=\left(A \cdot \sqrt{\frac{g}{a}} \cdot s h \sqrt{\frac{g}{a}} x+B \sqrt{\frac{g}{a}} \cdot \operatorname{ch} \sqrt{\frac{g}{a}} x\right)_{x=0}= \\
& =-Q \cdot \frac{1}{g}\left(1-\frac{1}{g \tau_{F}}\right) . \\
& \text { Then } B_{1}=-Q \cdot \sqrt{\frac{a}{g}} \cdot \frac{1}{g}\left(1-\frac{1}{g \cdot \tau_{T}}\right) .
\end{aligned}
$$

When $x=\delta$, we have: 


$$
\begin{aligned}
& T(\delta, g)=\left[A \cdot \sqrt{\frac{g}{a}} \cdot \operatorname{sh} \sqrt{\frac{g}{a}} \delta+B \sqrt{\frac{g}{a}} \cdot \operatorname{ch} \sqrt{\frac{g}{a}} \delta\right] \\
& =h\left[\frac{t_{\text {aver }}}{g}-\frac{t_{\text {aver }}}{g}-A \cdot \operatorname{ch} \sqrt{\frac{g}{a}} \delta-B \operatorname{sh} \sqrt{\frac{g}{a}} \delta\right]
\end{aligned}
$$

Hence $A$ is:

$$
A=-e \cdot \frac{M}{N}=Q \cdot \frac{1}{g} \cdot \sqrt{\frac{a}{g}}\left(1-\frac{1}{g \cdot \tau_{F}}\right) \cdot \frac{M}{N}
$$

where

$$
\begin{aligned}
& M=\left(\sqrt{\frac{g}{a}} \operatorname{ch} \sqrt{\frac{g}{a}} \delta+h \cdot s h \sqrt{\frac{g}{a}} \delta\right) ; \\
& N=\left(\sqrt{\frac{g}{a}} \operatorname{sh} \sqrt{\frac{g}{a}} \delta+h \cdot \operatorname{ch} \sqrt{\frac{g}{a}} \delta\right) .
\end{aligned}
$$
form:

Then complete solution for the image (6) has the following

$$
\begin{aligned}
& T(x, g)-\frac{t_{\text {aver }}}{g}=\frac{Q \cdot \sqrt{\frac{a}{g}}\left(g-\frac{1}{\tau}\right)}{g^{2}} \cdot \frac{M \cdot \operatorname{ch} \sqrt{\frac{g}{a}} x-N \cdot \operatorname{sh} \sqrt{\frac{g}{a}} x}{N}= \\
& =\frac{\Phi(g)}{g^{2} \cdot \varphi(g)}=\frac{\Phi(g)}{\Psi(g)}
\end{aligned}
$$

This expression (12) represents the ratio of two generalized polynomials with respect to $\mathrm{g}$, and the polynomial in the denominator does not contain a free term; all the conditions of the expansion theorem are satisfied.

We define the roots of polynomial $\Psi(g)$, for which we equate the denominator of equation (12) to zero:

$$
\begin{aligned}
& \Psi(g)=g^{2}\left(\sqrt{\frac{g}{a}} \cdot \operatorname{sh} \sqrt{\frac{g}{a}} \delta+h \cdot \operatorname{ch} \sqrt{\frac{g}{a}} \delta\right)= \\
& =g^{2}\left(-\frac{\mu}{\delta}+\operatorname{ctg} \mu\right)=0
\end{aligned}
$$

We get: 1) double root $g_{0}=0 ; 2$ ) innumerable number of roots $\left(g_{p}=-\frac{a}{\delta^{2}} \cdot \mu_{p}^{2}\right.$ ), determined from equation (13), in which we have:

$$
\mu=i \sqrt{\frac{g}{a}} \delta
$$

Finally, from equation (13), we have $\operatorname{ctg} \mu=\frac{\mu}{B i}$, where $B i=h \cdot \delta=\frac{\alpha}{\lambda} \cdot \delta$ - Biot number.

Using the expansion theorems of [6], we obtain:

$$
\begin{aligned}
& \lim _{g \rightarrow 0}\left\{\frac{d}{d g}\left[\frac{\Phi(g)}{\varphi(g)} \cdot e^{g \tau}\right]\right\}= \\
& =\lim _{g \rightarrow 0}\left[\tau \cdot e^{g \tau} \cdot \frac{\Phi(g)}{\varphi(g)}+e^{g \tau} \cdot \frac{\Phi^{\prime}(g)}{\varphi(g)}-e^{g \tau} \cdot \frac{\Phi(g) \cdot \varphi^{\prime}(g)}{[\varphi(g)]^{2}}\right]= \\
& =\tau \cdot \frac{Q}{\tau_{F} \cdot h}[h x-h \delta-1]+\frac{Q}{\tau_{F} \cdot h} \cdot \\
& {\left[(\delta-x)\left(h \tau_{F}-\frac{h \delta x}{2 a}\right)+\frac{h}{6 a}\left(\delta^{3}-x^{3}\right)+\frac{(x+\delta)^{2}}{2 a}+\tau_{F}\right]-} \\
& -\frac{Q}{\tau_{F} \cdot h} \cdot \frac{1}{h} \cdot[h x-h \delta-1] \cdot \frac{\delta(2-B i)}{2 a}= \\
& =\frac{Q}{\tau_{F} \cdot h}\left[(h x-h \delta-1)\left(\tau-\frac{\delta(2-B i)}{2 a h}\right)+\right. \\
& +(\delta-x)\left(h \tau_{T}-\frac{h \delta x}{2 a}\right)+\frac{h}{6 a}\left(\delta^{3}-x^{3}\right)+ \\
& \left.+\frac{(x-\delta)^{2}}{2 a}+\tau_{F}\right]
\end{aligned}
$$

where $\Phi(0)=\frac{Q}{\tau_{F}}[h x-h \delta-1]$;

$$
\begin{aligned}
& \Phi^{\prime}(0)=\frac{Q}{\tau}\left[(\delta-x)\left(h \tau_{F}-\frac{h \delta x}{2 a}\right)+\frac{h}{6 a}\left(\delta^{3}-x^{3}\right)+\right. \\
& \left.+\frac{(x+\delta)^{2}}{2 a}+\tau_{F}\right] \\
& \varphi(0)=h ; \varphi^{\prime}(0)=\frac{\delta}{2 a}(2-B i) .
\end{aligned}
$$

For other roots, we use the expansion theorem $[6,11,12]$ :

$L_{\tau}^{-1}\left[\frac{\Phi(g)}{\Psi(g)}\right]=\sum_{n=1}^{\infty} \frac{\Phi\left(g_{p}\right)}{\Psi^{\prime}\left(g_{p}\right)} \cdot e^{g_{n} \tau}$,

where $g_{p}$ - roots of polynomials.

For this purpose, we define $\Phi\left(g_{p}\right)$ and $\Psi\left(g_{p}\right)$ :

$$
\Phi\left(g_{p}\right)=-Q \cdot \frac{\sqrt{B i^{2}+\mu_{p}^{2}}}{\mu_{p}} \cdot\left(\frac{1}{\tau_{F}}+a \cdot \frac{\mu_{p}^{2}}{\delta^{2}}\right) \cdot \cos \mu_{p} \cdot \frac{x}{\delta}
$$




$$
\Psi^{\prime}\left(g_{p}\right)=\frac{a}{2} \cdot \frac{\mu_{p}^{3}}{\delta^{3}} \cdot \frac{B_{i}+B_{i}^{2}+\mu_{p}^{2}}{\sqrt{\mu_{p}^{2}+B i^{2}}}
$$

Then:

$$
\begin{aligned}
& \sum_{n=1}^{\infty} \frac{\Phi\left(g_{p}\right)}{\psi^{\prime}\left(g_{p}\right)} \cdot e^{g_{n} \tau}=-Q \delta \sum_{n=1}^{\infty} A_{p}\left(\frac{\delta^{2}}{a \cdot \tau_{F} \cdot \mu_{p}^{2}}+1\right) . \\
& \cos \mu_{p} \cdot \frac{x}{\delta} \cdot \exp \left(-\mu_{p}^{2} F_{o}\right)
\end{aligned}
$$

where $A_{p}=\frac{2\left(\mu_{p}^{2}+B_{i}^{2}\right)}{\mu_{p}^{2}\left(\mu_{p}^{2}+B i^{2}+B i\right)}$ - initial thermal amplitude; $F_{o}=\frac{a \tau}{\delta^{2}}$ - Fourier criterion (or dimensionless operating time of the friction unit).

The complete analytical equation determining the temperature field of friction nodes as a function of thickness of friction material $\delta$ and friction time $\tau$ has the following form:

$$
\begin{aligned}
& t(x, \tau)-t_{\text {aver }}=\frac{\left(1-\alpha_{H F .}\right) W_{H F .}}{A_{a} \cdot \tau_{F} \cdot \alpha}\left\{\frac { 2 } { \tau _ { F } } \left[(h x-h \delta-1)\left(\tau-\frac{\delta(2-B i)}{2 a h}\right)+\right.\right. \\
& \left.+(\delta-x)\left(h \tau_{F}-\frac{h \delta x}{2 a}\right)+\frac{h}{6 a}\left(\delta^{3}-x^{3}\right)+\frac{(x+\delta)^{2}}{2 a}+\tau_{F}\right]- \\
& -2 B i \sum_{n=1}^{\infty} A_{p}\left(\frac{\delta^{2}}{a \cdot \tau_{F} \cdot \mu_{p}^{2}}+1\right) \cdot \cos \mu_{n} \cdot \frac{x}{\delta} \\
& \cdot \exp \left(-\mu_{p}^{2} F_{o}\right)
\end{aligned}
$$

Then temperature in the zone of friction $(x=0)$ of brakes for the case of active heat dissipation takes the form:

$$
\begin{aligned}
& t(0, \tau)-t_{, m}=\frac{\left(1-\alpha_{H F}\right) W_{H F .}}{A_{a} \cdot \tau_{F} \cdot \alpha} \\
& \cdot\left[\tau_{N}(1+B i)+\frac{1}{F o}\left(2+\frac{2}{B i}-\frac{2}{3} B i\right) \cdot \frac{\tau}{\tau_{F}}-2 B i \sum_{n=1}^{\infty} A_{p} .\right. \\
& \left(1+\frac{\tau}{\tau_{F} \cdot F_{O} \cdot \mu_{p}^{2}}\right) \cdot \exp \left(-\mu_{p}^{2} F_{O}\right)
\end{aligned}
$$

The value of the temperature difference on the surface of brakes $(x=\delta)$, which determines heat dissipation (heat transfer), is accordingly equal to

$$
\begin{aligned}
& t(\delta, \tau)-t_{, m}=\frac{\left(1-\alpha_{H F}\right) W_{H F}}{A_{a} \cdot \tau_{F} \cdot \alpha} . \\
& \cdot\left[\tau_{N}+\frac{1}{F_{O}}\left(\frac{2}{B i}+3\right) \cdot \frac{\tau}{\tau_{F}}-2 B i \sum_{n=1}^{\infty} A_{p} .\right. \\
& \left.\left(1+\frac{\tau}{\tau_{T} \cdot F_{O} \cdot \mu_{p}^{2}}\right) \cdot \cos \mu_{p} \cdot \exp \left(-\mu_{p}^{2} F_{O}\right)\right] .
\end{aligned}
$$

The results of calculations of temperature modes have been used to estimate the temperature in the friction zone (19) and the temperature on the heat transfer surface (20) during bench and operational production tests. The effect of the maximum possible temperatures in the brake units has been determined by the choice of friction pairs (by their thermal stability) [9, 10].

From the analysis of equations (19), (20), characterizing temperature in the friction zone and the heat transfer surface, it is seen that the temperature field of friction pad is determined mainly by Biot number and Fourier criteria. The Fourier criterion characterizes the process of pure thermal conductivity of friction material for the $\mathrm{x}$ coordinate, and Biot number characterizes heat exchange of friction material with the surrounding medium.

In equation (20), which determines temperature difference during heat transfer of the friction unit, the last term of the sum is a convergent series, i.e. the algebraic sum of cosine waves with gradually damped oscillations at amplitude $A_{p}\left(1+\frac{\tau}{\tau_{T} \cdot F o \cdot \mu_{p}^{2}}\right) \cdot \exp \left(-F o \mu_{p}^{2}\right)$. The value of this thermal amplitude rapidly decreases both with increasing $\mu_{p}$, and with the passage of time (more precisely, Fo criterion). Consequently, the temperature field of friction material is a function of time, the distribution of which obeys the law of parabola. In case of very small values of the series (the last term), we observe the quasistationary regime for the field of the temperature gradient.

Consideration of the obtained temperature field of the friction unit provides a basis for studying the process of heat exchange with the environment, which takes place in two stages. In the first period of the thermal process, $0<\tau \leq \tau_{l}$, the depth of heated layer does not exceed reduced thickness of friction pad $\delta_{\text {red }}$, which can be determined from expression [7]:

$$
\delta_{\text {red }}=1,73 \sqrt{a_{\Phi . M .} \cdot \tau_{1}} .
$$

Equation (21) is characterized by criterion $F_{0}>0.333$ (which corresponds to $\approx 15-20$ seconds of friction unit operation and is acceptable, and thermal process in the first stage is determined by thermal conductivity in the friction pad $[13,14]$. 
When $\tau \geq \tau_{l}$, the temperature of the friction pad begins to vary throughout its volume simultaneously, i.e. the second stage of heating comes, which is also be characterized by heat exchange with the external medium according to the Newton's law of cooling:

$$
q=\alpha\left(t_{x=\delta}-t_{\text {aver }}\right) .
$$

In the process of heat transfer, with the same temperature difference, dissipation of specific heat flow can vary within a very wide range. This is explained by the fact that intensity of the heat transfer process, in addition to the temperature difference $\left(t_{x=\delta}-t_{\text {aver }}\right)$, depends on such factors as rotational speed of inertial brake masses, motion regime of the eat carrier (air), the thermophysical parameters of coolant, shape and dimensions of the friction unit, etc. The Newton-Richmann equation (22) does not take into account the influence of these factors on the heat transfer process and is externally characterized by a simple form. However, this equation includes the coefficient of heat transfer $\alpha$, and it is necessary to take into account all the factors that affect the heat transfer process for the correct determination of them. The value of heat transfer coefficient $\alpha$, taking into account the airflow cooling brake unit, has been determined from the empirical equation obtained as a result of our studies within the range of the Reynolds criterion $11760<R e_{a i r, l}<49400$ :

$$
\alpha=0,155 \frac{\lambda_{\text {air }}}{l} \cdot \operatorname{Re}_{\text {air }, e}^{0,745}=0,155 \cdot \frac{\lambda_{\text {air }}}{l}\left(\frac{w \cdot l}{v_{\text {air }}}\right)^{0,745}
$$

where $\alpha$ - coefficient of heat transfer of friction pairs of the brake unit, $\mathrm{W} / \mathrm{m}^{2}$. ${ }^{\circ} \mathrm{C}$; $\lambda_{\text {air }}$ - coefficient of thermal conductivity of the coolant (air), $\mathrm{W} / \mathrm{m} \cdot{ }^{\circ} \mathrm{C} ; l-$ typical geometric size, $\mathrm{m} ; w$ - average speed of air movement in the brake unit, $\mathrm{m}$; $v_{\text {air }}$ - coefficient of kinematic viscosity of air, $\mathrm{m}^{2} / \mathrm{s}$.

Blowing the friction unit with air leads to an increase in the coefficient of heat transfer $\alpha$, and, consequently, to a decrease in the temperature regime during friction of brakes friction pairs. The consequence of this is stabilization of the friction coefficient and a reduction of wear of the friction brakes, which ensures an increase in the serviceability of frictions pairs of brake units as a whole $[8,15]$.

\section{CONCLUSION}

The resulted calculation of a temperature mode of brake units allows designers and technologists to define optimum sizes and assemblage of brake units, to correctly select frictional material considering heat resistance. In case of necessity, using calculation of temperature regime of brake units, it is possible to provide measures for improvement in friction pairs of active heat dissipation. These measures ultimately improve reliability and durability of brake units, which ultimately leads to an increase in the passive safety of transport in general.

\section{REFERENCES}

[1] A.V. Chichinadze, Calculation and investigation of external friction during braking, Moscow: "Nauka" Publishing House, 1967, pp. 115147.

[2] V.A. Ditkin, P.I. Kuznetsov, Reference book on operational research, Moscow: State Publishing House of Technical and Theoretical Literature, 1951, pp. 198-237.

[3] G. Doetsch, Guide to the applications of Laplace transforms. Moscow: State Publishing House of Technical and Theoretical Literature, 1958, pp. 171-179.

[4] I.V. Kragelsky, Friction and wear, Moscow: Publishing house "Mechanical Engineering", 1962, pp. 41-44.

[5] A.V. Chichinadze, E.V. Zinoviev, To assessment of significance of certain determining parameters of friction during braking. Collection "Thermal dynamics of friction", Moscow: "Nauka" Publishing House, 1973, pp. 54-60.

[6] A.V. Lykov, Heat and mass transfer, Moscow, 1972, pp. 320-351.

[7] A.P. Begijanova, Temperature regime of belt brakes of caterpillar tractor. Moscow, 1968, pp. 39-41.

[8] F.K. Germanchuk, Durability and efficiency of braking devices, Moscow, 1973, pp. 97-141.

[9] E.A. Indikt, V.A. Cherniaykin, Operational reliability of trucks, Moscow, 1977, pp. 32-41.

[10] V.M. Kazarinov, Theoretical foundations of design and operation of auto brakes, Moscow, 1968, pp. 64-69.

[11] A.D. Kovalenko, Thermoelasticity, Kyiv, 1975, pp. 114-132.

[12] S.V. Belokurov, V.P. Belokurov, V.K. Zolnikov, O.N. Cherkasov "Methods of Multi-Criteria Optimization in Problems of Simulation of Trucking Industri”, Transportation Research Procedia, № 20, 2017, pp. 47-52.

[13] P. Kall, S.W. Wallance, Stochastic Programming, John Wiley and Sons, Chichester, 1994, pp. 247-254.

[14] J. Hibbis, Transport policy: The myth of integrated planning, London: The institute of economic affairs, 2000, pp. 66-69.

[15] X. Shen, X Yao, "Mathematical Modeling and Multiobjective Evolutionary Algorithms Applied to Dynamic Flexible Job Shop Scheduling Problems", Information Scienees, Vol. 298, 2015, pp. 198224. 